\title{
Association between expression of HOTAIR and invasiveness of gliomas, and its predictive value
}

\author{
Wei-Hua Zha0 ${ }^{1, B-D}$, Hong-Yu Yuan 2,B-D , Xiao-Yan Ren 2,B, , Kun Huang ${ }^{2, A, C, F}$, Zai-Yu Guo ${ }^{1, A, E, F}$ \\ ${ }^{1}$ Department of Neurosurgery, TEDA Hospital, Tianjin, China \\ $23^{\text {rd }}$ Department of Neurology, Heze Municipal Hospital, China \\ A - research concept and design; B - collection and/or assembly of data; C - data analysis and interpretation; \\ $D$ - writing the article; $E$ - critical revision of the article; $F$ - final approval of the article
}

\section{Address for correspondence \\ Kun Huang \\ E-mail: huangkun613123@163.com}

Funding sources

None declared

Conflict of interest

None declared

Received on August 31, 2018

Reviewed on September 21, 2018

Accepted on November 2, 2018

Published online on August 13, 2019

Cite as

Zhao WH, Yuan HY, Ren XY, Huang K, Guo ZY. Association between expression of HOTAIR and invasiveness of gliomas, and its predictive value. Adv Clin Exp Med. 2019;28(91):1179-1183. doi:10.17219/acem/99527

DOI

10.17219/acem/99527

\section{Copyright}

Copyright by Author(s)

This is an article distributed under the terms of the

Creative Commons Attribution Non-Commercial License

(http://creativecommons.org/licenses/by-nc-nd/4.0/)

\begin{abstract}
Background. Hox transcript antisense intergenic RNA (HOTAIR) is upregulated and associated with a poor prognosis in many cancer types. Besides, it is involved in the invasion and metastasis of non-small-cell lung cancer and nasopharyngeal carcinoma.
\end{abstract}

Objectives. The aim of this study was to investigate the association between the expression of HOTAIR and the grades of gliomas, and to explore its possible mechanism, as well as to evaluate the value of HOTAIR applied in predicting the grades of gliomas.

Material and methods. A total of 123 patients undergoing glioma surgeries were enrolled. Patients with grade I and grade II-IV tumors were regarded as the control group $(n=36)$ and the case group $(n=87)$, respectively. The expression of HOTAIR, matrix metalloproteinase 7 (MMP-7), matrix metalloproteinase 9 (MMP-9), and vascular endothelial growth factor (VEGF) was detected with quantitative reverse transcriptionpolymerase chain reaction (qRT-PCR) in glioma tissues and then compared between grade I and grades II-IV. The correlation between the relative expression of HOTAIR and that of MMP-7, MMP-9 and VEGF was analyzed. Multivariate analysis was performed to identify independent risk factors. Receiver operating characteristic (ROC) curve was employed to evaluate the predictive value.

Results. The relative expression of HOTAIR, MMP-7, MMP-9, and VEGF was lower in glioma tissues of grade I than in the case of grades $\|-I V$, and the relative expression of HOTAIR was positively correlated with the relative expression of MMP-7, MMP-9 and VEGF. Multivariate analysis showed that the relative expression of HOTAIR was independently associated with the grades of gliomas, but the relative expression of MMP-7, MMP-9 and VEGF was not. Besides, multivariate analysis showed that the expression level of HOTAIR $>0.40$ was an independent risk factor for grades II-IV after classifying the relative expression of HOTAIR, and ROC analysis showed that the expression level of HOTAIR $>0.40$ had a moderate value when applied in predicting grades II-IV.

Conclusions. Hox transcript antisense intergenic RNA might promote the invasion of gliomas through upregulating the expression of MMP-7, MMP-9 and VEGF, and the expression level of HOTAIR $>0.40$ had a moderate value when applied in predicting grades $\|-\mid \mathrm{V}$.

Key words: gliomas, hox transcript antisense intergenic RNA, grades, predictive value, invasiveness 


\section{Introduction}

As the most common primary tumors in the central nervous system among adults, ${ }^{1,2}$ gliomas are classified into 4 grades that reflect the degree of invasiveness/malignancy according to the current 2016 World Health Organization (WHO) Classification of Tumours of the Central Nervous System. ${ }^{3}$ Grade I refers to benign and more circumscribed tumors with low proliferative potential. Grade II-IV tumors are malignant and diffusely infiltrative with increased cellular abnormalities, and are referred to as diffuse gliomas because of the characteristic of extensive, diffuse infiltration of glioma cells into the brain tissue. ${ }^{4}$

Hox transcript antisense intergenic RNA (HOTAIR) belongs to long non-coding RNAs (lncRNAs). Many studies have indicated that lncRNAs can be applied in predicting the prognosis in various cancer types. ${ }^{5-8}$ Hox transcript antisense intergenic RNA is the first lncRNA discovered to have a trans-repressive role. ${ }^{9,10}$ It is upregulated and associated with a poor prognosis in many cancer types. ${ }^{11-15}$ Apart from that, it is involved in the invasion and metastasis of non-small-cell lung cancer and nasopharyngeal carcinoma through modulating the expression of matrix metalloproteinase 7 (MMP-7), matrix metalloproteinase 9 (MMP-9) and vascular endothelial growth factor (VEGF). ${ }^{16,17}$ In this paper, the association between the relative expression of HOTAIR and the grades of gliomas was analyzed, and the possible mechanism was explored; moreover, the value of HOTAIR applied in predicting the grades of gliomas was evaluated. The aim of the study was to determine the potential of HOTAIR as a biomarker of invasiveness for gliomas.

\section{Material and methods}

\section{Patients}

A total of 123 patients undergoing glioma surgery were enrolled in Heze Municipal Hospital, China, from January 2013 to January 2017. There were 70 males and 53 females among them, and their average age was $44.76 \pm 15.82$ years. A definite diagnosis of gliomas was made through a histopathologic examination in all patients. Neither radiotherapy nor chemotherapy were performed before operation. According to the current 2016 WHO Classification of Tumours of the Central Nervous System, grades I, II, III, and IV were found in 36, 32, 33, and 22 patients, respectively. The patients with grade I and grade II-IV tumors were regarded as the control group $(n=36)$ and the case group $(n=87)$, respectively. Immediately after excision, glioma tissue samples were placed in liquid nitrogen for $10 \mathrm{~min}$, and then stored in a refrigerator at a temperature of $-80^{\circ} \mathrm{C}$. This study received the approval of the ethics committee of Heze Municipal Hospital (approval No. hz201216039). All patients provided informed consent.

\section{RNA extraction and quantitative reverse transcription-polymerase chain reaction}

The total RNA from glioma tissues was extracted with the TRIzol ${ }^{\mathrm{TM}}$ reagent (Ambion, Cat. No. 15596-026; Invitrogen, Carlsbad, USA) according to the manufacturer's instructions. The complementary DNA (cDNA) was synthesized with the First Strand cDNA Synthesis kit (Novagen, Cat. No. 69001-3; Merck \& Co., Inc., Kenilworth, USA). The primer sequences for HOTAIR, MMP-7, MMP-9 and VEGF were 5'-GAGAACGCTGGAAAAACCTG-3' (forward) and 5'-GTCAGAAAATGCTTCCCCAA-3' (reverse), 5'-TACAGGATCATTGGCTACACACC-3' (forward) and 5'-GGTCACATCGCTCCAGACT-3' (reverse), 5'-TGTACCGCTATGGTTACACTCG-3' (forward) and 5'-GGCAGGGACAGTTGCTTCT-3' (reverse), and 5'-AGGGCAGAATCATCACGAAGT-3' (forward) and 5'AGGGTCTCGATTGGATGGCA-3' (reverse), respectively. GADPH was regarded as the reference gene (forward primer: 5'-AGGTCCACCACTGACACGTT-3', and reverse primer: 5'-GCCTCAAGATCATCAGCAAT-3'). The amplification conditions were as follows: $94^{\circ} \mathrm{C}$ for $3 \mathrm{~min}$; 40 cycles of $94^{\circ} \mathrm{C}$ for $30 \mathrm{~s}, 55^{\circ} \mathrm{C}$ for $30 \mathrm{~s}$ and $72^{\circ} \mathrm{C}$ for $1 \mathrm{~min}$; and $72^{\circ} \mathrm{C}$ for $7 \mathrm{~min}$. The quantitative reverse transcriptionpolymerase chain reaction (qRT-PCR), data acquisition and analysis were performed with the ABI 7500 system (Life Technologies, Grand Island, USA). The relative expression of target genes was evaluated with the $2^{-\Delta \Delta C t}$ method.

\section{Statistical analysis}

Statistical analysis was carried out using the IBM SPSS Statistics v. 21.0 for Windows (IBM Inc., Armonk, USA), and significance was set at $\mathrm{p}<0.05$. The distribution of data was determined with the KolmogorovSmirnov test. Normally distributed data was expressed as mean \pm standard deviation (SD) and compared with Student's t-test between glioma grade I and grades II-IV. Simple linear correlation analysis was employed to analyze the correlation between normally distributed data. In order to analyze the association between the expression levels of HOTAIR and the grades of gliomas, the relative expression of HOTAIR was classified into 4 levels based on the quartiles of grade I (level 1: $\leq \mathrm{Q} 1$; level 2: >Q1 and $\leq$ Q2; level 3: $>$ Q2 and $\leq$ Q3; level 4: $>$ Q3). Univariate analysis was performed with a simple $\chi^{2}$ test. Multivariate analysis was then performed with a backward stepwise logistic regression model. Receiver operating characteristic (ROC) curve was employed to evaluate the predictive value of independent risk factors. 


\section{Results}

\section{Relative expression of HOTAIR}

The relative expression of HOTAIR in glioma tissues of grade I $(n=36)$ and grades II-IV $(n=87)$ was $0.41 \pm 0.20$ and $0.80 \pm 0.31$, respectively. The difference was statistically significant $(\mathrm{p}<0.001)$.

\section{Relative expression of MMP-7, MMP-9 and VEGF}

The relative expression of MMP-7, MMP-9 and VEGF in glioma tissues of grade I was $0.35 \pm 0.17,0.31 \pm 0.19$ and $0.39 \pm 0.21$, respectively, all of which were significantly lower compared to grades II-IV $(0.79 \pm 0.26,0.70 \pm 0.25$ and $0.83 \pm 0.35$, respectively) (all $\mathrm{p}<0.001$ ).

\section{Simple linear correlation analysis between relative expression of HOTAIR and relative expression of MMP-7, MMP-9 and VEGF}

Simple linear correlation analysis showed that the relative expression of HOTAIR was positively correlated with the relative expression of MMP-7, MMP-9 and VEGF $(\mathrm{r}=0.782,0.791$ and 0.778 , respectively; all $\mathrm{p}<0.05)$ in all 123 glioma patients.

\section{Association between relative expression of HOTAIR, MMP-7, MMP-9 and VEGF, and grades of gliomas}

Multivariate analysis showed that the relative expression of HOTAIR was independently associated with the grades of gliomas after adjusting sex and age, but the relative expression of MMP-7, MMP-9 and VEGF was not independently associated with the grades of gliomas (Table 1). High expression of HOTAIR was an independent risk factor for grades II-IV.

\section{Association between expression levels of HOTAIR and grades of gliomas}

The classifying and univariate analysis results are shown in Table 2. Multivariate analysis showed that the expression level of HOTAIR >Q2 (0.40) was an independent risk factor for grades II-IV after adjusting sex and age (Table 3). The odds ratios (ORs) of level 3 and 4 were 5.061 and 9.016, respectively.

\section{Predictive value of HOTAIR expression level}

The results of the expression level of HOTAIR >Q2 (0.40) applied in predicting grades II-IV are shown in Table 4. The area under ROC curve (AUC) was 0.716 (standard error: 0.056; $\mathrm{p}<0.001)$, which indicated that the expression level of HOTAIR >Q2 (0.40) had a moderate value when applied in predicting grades II-IV.

\section{Discussion}

Since HOTAIR is firstly discovered in human fibroblasts ${ }^{18}$ many studies have reported that HOTAIR is significantly upregulated in many cancer types and play an important role in their development. For gliomas, HOTAIR can act as a prognostic factor for the survival

Table 1. Association between relative expression of HOTAIR, MMP-7, MMP-9 and VEGF, and grades of gliomas after adjusting sex and age

\begin{tabular}{|l|c|c|c|c|c|c|}
\hline \multicolumn{1}{|c|}{ Genes } & $\begin{array}{c}\text { Regression } \\
\text { coefficient }\end{array}$ & Standard error & Wald & OR & 95\% Cl \\
\hline HOTAIR & 0.421 & 0.184 & 6.597 & 4.372 & $1.286-7.958$ \\
\hline MMP-7 & 0.283 & 0.122 & 1.674 & 1.984 & 0.029 \\
\hline MMP-9 & 0.276 & 0.117 & 1.582 & 1.886 & $0.783-4.141$ \\
\hline VEGF & 0.291 & 0.124 & 1.785 & 2.094 & $0.754-3.908$ \\
\hline
\end{tabular}

HOTAIR - hox transcript antisense intergenic RNA; MMP-7 - matrix metalloproteinase-7; MMP-9 - matrix metalloproteinase-9; VEGF - vascular endothelial growth factor; OR - odds ratio; Cl - confidence interval; Wald - the Wald $x^{2}$ test. Grading of gliomas was regarded as the dependent variable (binary variable: grade II-IV was assigned to 1, and grade I to 0), and the relative expression of HOTAIR, MMP-7, MMP-9, and VEGF (quantitative variables), age (quantitative variable) and sex (binary variable) were regarded as the independent variables.

Table 2. Classifying and univariate analysis (simple $x^{2}$ test) results of relative expression of HOTAIR

\begin{tabular}{|c|c|c|c|c|}
\hline Relative expression of HOTAIR & $\begin{array}{l}\text { Grades II-IV } \\
\qquad(\mathrm{n}=87)\end{array}$ & $\begin{array}{l}\text { Grade I } \\
(n=36)\end{array}$ & $x^{2}$ & $p$-value \\
\hline Level 1: sQ1 (0.19) & 2 & 9 & \multirow{4}{*}{32.379} & \multirow{4}{*}{$<0.001$} \\
\hline Level 2: >Q1 and sQ2 (0.40) & 4 & 9 & & \\
\hline Level 3: >Q2 and $\leq \mathrm{Q} 3(0.58)$ & 19 & 9 & & \\
\hline Level 4: >Q3 & 62 & 9 & & \\
\hline
\end{tabular}

Q1-Q3 - quartiles of grade I. 
Table 3. Association between expression levels of HOTAIR and grades of gliomas after adjusting sex and age

\begin{tabular}{|l|c|c|c|c|c|c|}
\multicolumn{1}{c|}{$\begin{array}{c}\text { Expression levels } \\
\text { of HOTAIR }\end{array}$} & $\begin{array}{c}\text { Regression } \\
\text { coefficient }\end{array}$ & Standard error & Wald & OR & $95 \% \mathrm{Cl}$ & $\mathrm{p}$-value \\
\hline Level 1 & - & - & 7.325 & - & - & 0.009 \\
\hline Level 2 & 0.412 & 0.283 & 1.584 & 1.575 & $0.332-3.658$ & 0.274 \\
\hline Level 3 & 0.476 & 0.315 & 6.278 & 5.061 & $1.158-7.029$ & 0.032 \\
\hline Level 4 & 0.511 & 0.367 & 18.213 & 9.016 & $1.392-12.164$ & $<0.001$ \\
\hline
\end{tabular}

Grading of gliomas was regarded as the dependent variable (binary variable: grade II-IV was assigned to 1, and grade I to 0), and the relative expression of HOTAIR (rank variable), age (quantitative variable) and sex (binary variable) were regarded as the independent variables. Level 1 was regarded as the reference.

Table 4. Results of the expression level of HOTAIR >Q2 (0.40) applied in predicting grade II-IV

\begin{tabular}{|c|c|c|c|}
$\begin{array}{c}\text { Cut-off } \\
\text { value }\end{array}$ & $\begin{array}{c}\text { Predicting } \\
\text { criterion }\end{array}$ & $\begin{array}{c}\text { Grade II-IV } \\
(\mathrm{n}=87)\end{array}$ & $\begin{array}{c}\text { Grade I } \\
(\mathrm{n}=36)\end{array}$ \\
\hline Q2 (0.40) & $>0.40$ (positive) & 81 & 18 \\
\hline & $\leq 0.40$ (negative) & 6 & 18 \\
\hline
\end{tabular}

of glioma patients and a biomarker for the identification of glioma molecular subtypes. ${ }^{11}$ In vitro, HOTAIR is associated with the progression of the cell cycle of glioma cells. Downregulation of HOTAIR can inhibit the proliferation, invasion and migration of tumor cells, and meanwhile promote the apoptosis of glioma cells. ${ }^{2,19}$ In our study, the relative expression of HOTAIR was lower in glioma tissues of grade I than in the case of grades II-IV, and multivariate analysis showed that high expression of HOTAIR (>0.40) was an independent risk factor for grades II-IV. These results suggested that HOTAIR was associated with the grades of gliomas and could act as a biomarker reflecting the invasiveness of gliomas.

Hox transcript antisense intergenic RNA is involved in the Wnt/ $\beta$-catenin, Akt and p53 signaling pathways through the combination with polycomb repressive complex 2 (PRC2) in tumor cells, ${ }^{14}$ which may affect the invasion and migration of tumor cells. ${ }^{20}$ The Wnt/ $\beta$-catenin signaling pathway plays an important role in the development of tumors. ${ }^{21}$ HOTAIR can activate the Wnt/ $\beta$ catenin signaling pathway through downregulating the expression of Wnt inhibitory factor 1 (WIF-1), ${ }^{22}$ which may reduce the degradation of $\beta$-catenin in the cytoplasm. ${ }^{23}$ $\beta$-catenin can upregulate the expression of VEGF, MMP-7 and cylinD1 as a transcriptional factor after the entry into the nucleus. Vascular endothelial growth factor, MMP-7 and cylinD1 may induce the formation of tumor vessels, and promote the invasion and migration of tumor cells.

As one of important tumor suppressor genes in the Akt signaling pathway, the phosphatase and tensin homology deleted on chromosome 10 (PTEN) gene can inhibit the activation of the Akt signaling pathway. Hox transcript antisense intergenic RNA may silence PTEN through enhancing the promoter methylation of PTEN, which may lead to the activation of the Akt signaling pathway. The activation of the Akt signaling pathway can promote the invasion and migration of tumor cells, and meanwhile reduce the apoptosis of tumor cells by upregulating the expression of MMP-9 and downregulating the expression of BAX and FOXO1. ${ }^{12}$ Besides, HOTAIR may induce the formation of tumor vessels by upregulating the expression of VEGF and downregulating the expression of transforming growth factor $\beta$ (TGF- $\beta$ ) via the p53 signaling pathway. ${ }^{24}$

In this study, the relative expression of HOTAIR, MMP-7, MMP-9, and VEGF was lower in glioma tissues of grade I than in the case of grades II-IV, and the relative expression of HOTAIR was positively correlated with the relative expression of MMP-7, MMP-9 and VEGF. However, multivariate analysis showed that the relative expression of MMP-7, MMP-9 and VEGF was not independently associated with the grades of gliomas. These results suggested that high expression of MMP-7, MMP-9 and VEGF might be induced by HOTAIR in grade II-IV tumors. Therefore, HOTAIR might promote the invasion of gliomas through upregulating the expression of MMP-7, MMP-9 and VEGF. Next, the value of the expression level of HOTAIR $>0.40$ applied in predicting grades II-IV was evaluated with ROC curve. This result showed that the expression level of HOTAIR $>0.40$ had a moderate value when applied in predicting grades II-IV.

\section{Conclusions}

Hox transcript antisense intergenic RNA might promote the invasion of gliomas through upregulating the expression of MMP-7, MMP-9 and VEGF, and the expression level of HOTAIR $>0.40$ had a moderate value when applied in predicting grades II-IV.

\section{ORCID iDs}

Wei-Hua Zhao (D) https://orcid.org/0000-0002-0258-1860 Hong-Yu Yuan (D) https://orcid.org/0000-0001-9749-514X Xiao-Yan Ren (D) https://orcid.org/0000-0003-0003-7156 Kun Huang (D) https://orcid.org/0000-0003-2916-0596 Zai-Yu Guo (1) https://orcid.org/0000-0002-1359-8262

\section{References}

1. Morgan LL. The epidemiology of glioma in adults: A "state of the science" review. Neuro Oncol. 2015;17(4):623-624.

2. Chen Y, Bian Y, Zhao S, et al. Suppression of PDCD4 mediated by the long non-coding RNA HOTAIR inhibits the proliferation and invasion of glioma cells. Oncol Lett. 2016;12(6):5170-5176.

3. Louis DN, Perry A, Reifenberger G, et al. The 2016 World Health Organization Classification of Tumours of the Central Nervous System: A summary. Acta Neuropathol. 2016;131(6):803-820. 
4. Alfonso JCL, Talkenberger K, Seifert M, et al. The biology and mathematical modelling of glioma invasion: A review. J $R$ Soc Interface. 2017;14(136):20170490.

5. Gong Z, Zhang S, Zhang W, et al. Long non-coding RNAs in cancer. Sci China Life Sci. 2012;55(12):1120-1124.

6. Zhang W, Huang C, Gong Z, et al. Expression of LINC00312, a long intergenic non-coding RNA, is negatively correlated with tumor size but positively correlated with lymph node metastasis in nasopharyngeal carcinoma. J Mol Histol. 2013;44(5):545-554.

7. Tang $\mathrm{K}$, Wei $\mathrm{F}$, Bo $\mathrm{H}$, et al. Cloning and functional characterization of a novel long non-coding RNA gene associated with hepatocellular carcinoma. Prog Biochem Biophys. 2014;41(2):153-162.

8. Gong Z, Zhang S, Zeng Z, et al. LOC401317, a p53-regulated long non-coding RNA, inhibits cell proliferation and induces apoptosis in the nasopharyngeal carcinoma cell line HNE2. PLoS One. 2014;9(11): e110674.

9. Shah N, Sukumar S. The Hox genes and their roles in oncogenesis. Nat Rev Cancer. 2010;10(5):361-371.

10. Wan Y, Chang HY. HOTAIR: Flight of noncoding RNAs in cancer metastasis. Cell Cycle. 2010;9(17):3391-3392.

11. Zhang JX, Han L, Bao ZS, et al. HOTAIR, a cell cycle-associated long noncoding RNA and a strong predictor of survival, is preferentially expressed in classical and mesenchymal glioma. Neuro Oncol. 2013; 15(12):1595-1603.

12. Li D, Feng J, Wu T, et al. Long intergenic noncoding RNA HOTAIR is overexpressed and regulates PTEN methylation in laryngeal squamous cell carcinoma. Am J Pathol. 2013;182(1):64-70.

13. Kogo R, Shimamura T, Mimori K, et al. Long noncoding RNA HOTAIR regulates polycomb-dependent chromatin modification and is associated with poor prognosis in colorectal cancers. Cancer Res. 2011; 71(20):6320-6326.
14. Gupta RA, Shah N, Wang KC, et al. Long non-coding RNA HOTAIR reprograms chromatin state to promote cancer metastasis. Nature. 2010;464(7291):1071-1076.

15. Xu C, Yang M, Tian J, et al. MALAT-1: A long non-coding RNA and its important $3^{\prime}$ end functional motif in colorectal cancer metastasis. Int J Oncol. 2011;39(1):169-175.

16. Nie Y, Liu X, Qu S, et al. Long non-coding RNA HOTAIR is an independent prognostic marker for nasopharyngeal carcinoma progression and survival. Cancer Sci. 2013;104(4):458-464.

17. Liu XH, Liu ZL, Sun M, et al. The long non-coding RNA HOTAIR indicates a poor prognosis and promotes metastasis in non-small cell lung cancer. BMC Cancer. 2013;13:464.

18. Rinn JL, Kertesz M, Wang JK, et al. Functional demarcation of active and silent chromatin domains in human HOX loci by noncoding RNAs. Cell. 2007;129(7):1311-1323.

19. Yang $B$, Wei $Z Y$, Wang $B Q$, et al. Down-regulation of the long noncoding RNA-HOX transcript antisense intergenic RNA inhibits the occurrence and progression of glioma. J Cell Biochem. 2018;119(2):2278-2287.

20. Hanahan D, Weinberg RA. Hallmarks of cancer: The next generation. Cell. 2011;144(5):646-674.

21. Zeng ZY, Zhou YH, Zhang WL, et al. Gene expression profiling of nasopharyngeal carcinoma reveals the abnormally regulated Wnt signaling pathway. Hum Pathol. 2007;38(1):120-133.

22. MacDonald BT, Tamai K, He X. Wnt/beta-catenin signaling: Components, mechanisms, and diseases. Dev Cell. 2009;17(1):9-26.

23. Ying Y, Tao Q. Epigenetic disruption of the WNT/beta-catenin signaling pathway in human cancers. Epigenetics. 2009;4(5):307-312.

24. Teodoro JG, Evans SK, Green MR. Inhibition of tumor angiogenesis by p53: A new role for the guardian of the genome. J Mol Med (Berl). 2007;85(11):1175-1186. 